\title{
Ultrafast laser and amplifier sources
}

\author{
A. Rundquist ${ }^{1}$, C. Durfee ${ }^{1}$, Z. Chang ${ }^{1}$, G. Taft ${ }^{1}$, E. Zeek ${ }^{1}$, S. Backus ${ }^{1}$, M.M. Murnane ${ }^{1}$, H.C. Kapteyn ${ }^{1}$, I. Christov ${ }^{2}$, \\ V. Stoev ${ }^{2}$ \\ ${ }^{1}$ Center for Ultrafast Optical Science, University of Michigan, Ann Arbor, MI 48109-2099, USA \\ (Fax: +313/763-4876; E-mail: kapteyn@umich.edu) \\ 2 Department of Physics, Sofia University, Sofia, Bulgaria
}

Received: 28 April 1997/Revised version: 15 May 1997

\begin{abstract}
There has been remarkable progress in the development of high peak-power ultrafast lasers in recent years. Lasers capable of generating terawatt peak powers with unprecedented short pulse durations can now be built on a single optical table in a small laboratory. The rapid technological progress has made possible a host of new scientific advances in high-field science, such as the generation of coherent femtosecond X-ray pulses, and the generation of MeV-energy electron beams and high-energy ions. In this paper, we review progress in the development and design of ultrafast highpower lasers based on Ti:sapphire, including the ultrafast laser oscillators that are a very important enabling technology for high-power ultrafast systems, and ultrafast amplified laser systems that generate 20 fs duration pulses with several watts average power at kilohertz repetition-rates. Ultrafast waveform measurements of these pulses demonstrate that such short pulses can be generated with high fidelity. Finally, we discuss applications of ultrafast high-power pulses, including the generation of femtosecond to attosecond X-ray pulses.
\end{abstract}

The 1990s have seen rapid progress in ultrafast laser technology [1,2]. Ultrafast light sources today are "turn-key" devices, producing peak output powers on the order of a megawatt with pulse durations under $10 \mathrm{fs}$, directly from a simple laser [3-8]. Since an optical cycle period in the visible and near-infrared is $2-3 \mathrm{fs}$, this pulse duration approaches fundamental limits for devices operating in this wavelength range. Important new measurement techniques [9] have also been developed, which can extract the complete waveform of an optical pulse of only a few cycles in duration $[10,11]$. Such precise measurement tools are essential to properly characterize and utilize extremely short optical pulses.

A major application of ultrafast lasers is to study the interaction of atoms, molecules, and plasmas with intense light. These high-field science applications require peak power in the range of $10^{10}-10^{15} \mathrm{~W}$. Such high-power short pulses are generated using laser amplifier systems. The technology for generating high-power amplified ultrafast pulses has progressed very rapidly over the past decade [12-22]. Amplification of the energy in an ultrashort pulse by factors of $10^{7}$ or more is now routinely achieved, resulting in a peak power of in the terawatt range from small-scale lasers, and of up to a petawatt from larger systems. By focusing these high-power optical pulses, light intensities of greater than $10^{20} \mathrm{~W} \mathrm{~cm}^{-2}$ can be generated, which correspond to an intensity greater than that which would be obtained by focusing the entire solar flux incident on the earth onto a pin-head. Because the size of a laser depends primarily on its pulse energy, by reducing the duration of the pulse being amplified, we can achieve extremely-high power densities using lasers of a realistic and compact scale. As a result, by reducing the pulse duration to $10-20 \mathrm{fs}$, even very small-scale laboratory lasers operating at kilohertz repetition rates $[23,24]$ are now easily capable of generating light intensities corresponding to an electric field in excess of that binding a valence electron to an atom.

Remarkable progress in high-field optical science has followed as a direct result of this technological progress. It is now possible to experimentally investigate highly-nonlinear processes in atomic, molecular, plasma, and solid-state physics, and to access previously-unexplored states of matter. Ultrashort-pulses at ultraviolet and soft X-ray wavelengths can be generated through harmonic upconversion [25-28] and also at hard and soft X-ray wavelengths through the creation of an ultrafast laser-produced plasma [29] or by scattering off electron beams [30-32]. Such ultrafast soft- and hard-X-ray pulses can be used to directly probe both longand short-range atomic dynamics and to monitor the evolution of highly-excited systems [33]. In other work, the use of extremely short-pulse high-intensity lasers may make it possible to generate coherent $\mathrm{X}$-rays with pulse duration as short as $10^{-16}$ seconds (or 100 attoseconds) [34-36].

\section{Ultrashort-pulse sources}

The technology of femtosecond lasers changed dramatically with the demonstration in 1990 of the self-modelocked Ti:sapphire laser by Sibbett and his group [1]. Titaniumdoped sapphire is a solid-state laser material with extreme- 


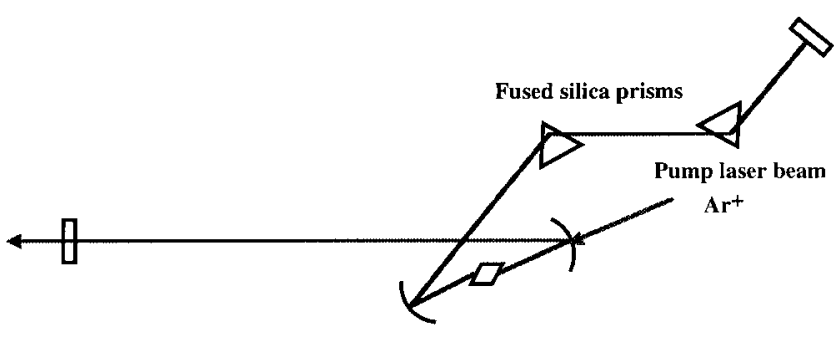

Fig. 1. Schematic diagram of a self-modelocked Ti:sapphire laser

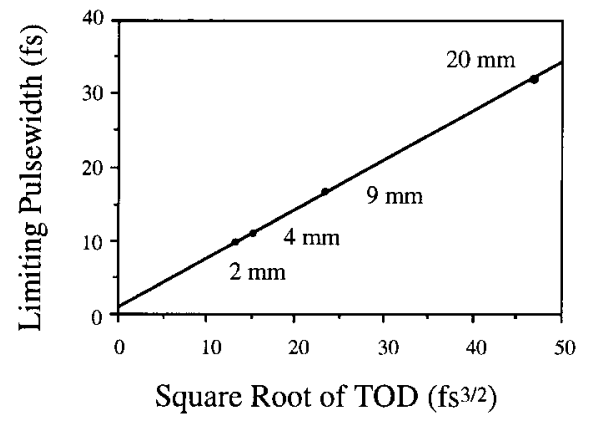

Fig. 2. Shortest pulse width vs. the square root of TOD in a Ti:sapphire laser (at $800 \mathrm{~nm}$ )

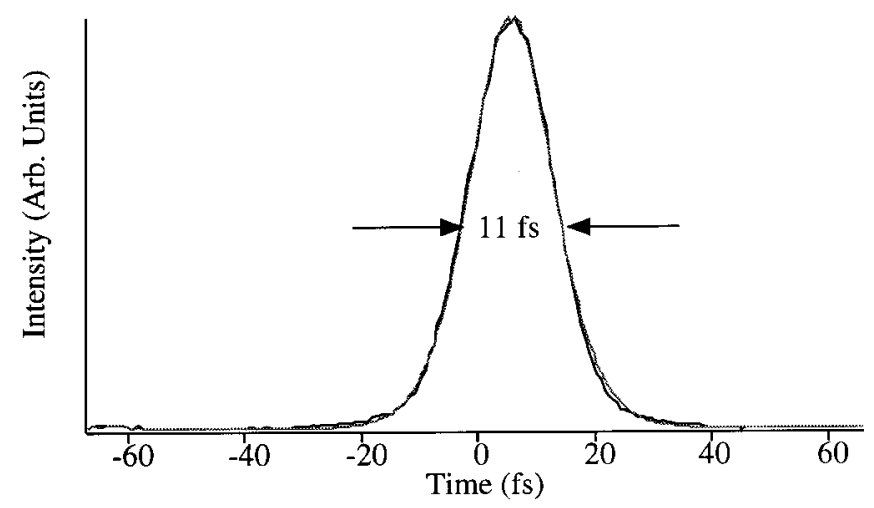

Fig. 3. Autocorrelation of an $11 \mathrm{fs}$ pulse from a TOD-limited Ti:sapphire laser

ly desirable properties [37,38]: a gain bandwidth spanning the wavelength region from $700 \mathrm{~nm}$ to $1100 \mathrm{~nm}$, very high thermal conductivity, and an energy storage density approaching $1 \mathrm{~J} / \mathrm{cm}^{2}$. This last property, although desirable for high-energy amplification, was thought to prohibit the use of Ti:sapphire in femtosecond modelocked lasers. Existing passively mode-locked dye lasers [39] relied on the low-energy storage density of the laser dye to facilitate the mode-locking process. Thus passive modelocking is not feasible using most solid-state gain media. However, the selfmodelocked Ti:sapphire laser relies on a different and very universal mechanism to facilitate short-pulse generation: the Kerr nonlinearity of the laser crystal. Since this nonlinearity is instantaneous, and independent of the energy storage density of the laser medium, it makes possible an entirely new class of reliable, high average power, ultrashort-pulse lasers based on Kerr-lens modelocking. The resulting laser configuration is attractive in its simplicity, as illustrated in Fig. 1. The gain medium is sandwiched between two focusing mirrors to re- duce the required area of the pump beam. A flat mirror and output coupler complete the laser cavity, with the only other addition being a prism pair to compensate dispersion.

The most significant advance in Ti:sapphire oscillator design since its original demonstration has been a dramatic reduction in achievable pulse duration. This was accomplished in work of our group and others [2,3,5-7, 10, 4049] by reducing overall dispersion in the laser. This can be done by using physically shorter Ti:sapphire crystals and optimum prism materials, or special mirrors [46]. Dispersion is extremely important in femtosecond lasers. It stabilizes the pulse in the laser and also determines the limiting pulsewidth for a given design geometry. For long-pulse (narrow-bandwidth) operation, Kerr-lens modelocked lasers work in a negative-dispersion regime [50,51]. The lasing bandwidth and pulsewidth can be controlled by adjusting the amount of negative dispersion, i.e., by changing the amount of glass in the laser by adjusting an intracavity prism.

In order to generate the shortest pulses, dispersion in the laser must be reduced to near zero (with a small amount of net-negative dispersion needed to compensate for self-phase modulation in the laser medium). In other words, the roundtrip time (or group delay) for light in the laser cavity must be nearly frequency independent, i.e., $T(\omega)=\delta \phi / \delta \omega=T_{0} \approx$ const., where $\phi$ is the total phase advance of light after one cavity round-trip. $T(\omega)$ can be expressed as a Taylor series about the center frequency $\omega_{0}$

$$
\begin{aligned}
T(\omega)=\frac{\partial \phi}{\partial \omega} & =\phi^{\prime}\left(\omega_{0}\right)+\phi^{\prime \prime}\left(\omega_{0}\right) \Delta \omega \\
& +\frac{1}{2} \phi^{\prime \prime \prime}\left(\omega_{0}\right) \Delta \omega^{2}+\frac{1}{6} \phi^{\prime \prime \prime \prime}\left(\omega_{0}\right) \Delta \omega^{3}+\ldots,
\end{aligned}
$$

where $\phi^{\prime}, \phi^{\prime \prime}$, etc. are the derivatives of the phase with respect to frequency. From the above we see that the second-order $\left(\phi^{\prime \prime}\right)$ dispersion term (also called group velocity dispersion or GVD) leads to a linear chirp. This GVD term can be adjusted by adjusting the amount of prism glass in the laser, and the separation of the prism pair. The next term, proportional to $\phi^{\prime \prime \prime}$, is the third-order dispersion (TOD) or quadratic chirp term, while the next term again is the fourth-order dispersion (4OD) or cubic chirp term.

Our past work demonstrated that the shortest pulsewidth for a given laser set-up is determined by the presence of higher-order dispersion in the laser, which prevents frequencies far from the line center from being modelocked. This is illustrated in Fig. 2, which plots the shortest pulse duration obtained from a laser as a function of the net TOD (the largest non-cancelable term) of the laser. In this series of experiments [3, 5, 40,41], we reduced the TOD in a Ti:sapphire laser by reducing both the material in the cavity, and choosing the prism glass type to minimize TOD. Reducing the crystal length from $2 \mathrm{~cm}$ (with LaFN28 prisms), to $9 \mathrm{~mm}$ (with LaKL21 prisms), then $4.5 \mathrm{~mm}$ (with fused silica prisms), and finally $2 \mathrm{~mm}$ (again with fused silica prisms) makes it possible to minimize TOD in the laser and thus decrease the minimum pulsewidth from $32 \mathrm{fs}$ to $17 \mathrm{fs}$, and finally $<10 \mathrm{fs}$. Figure 3 shows the autocorrelation of an $11 \mathrm{fs}$ pulse obtained from the most widely implemented laser configuration, which uses a $4.5 \mathrm{~mm}$ laser crystal. The initial design of Sibbet, designed without regard to TOD, obtained $60-100 \mathrm{fs}$ pulse duration. Figure 4 illustrates how proper choice of prism ma- 


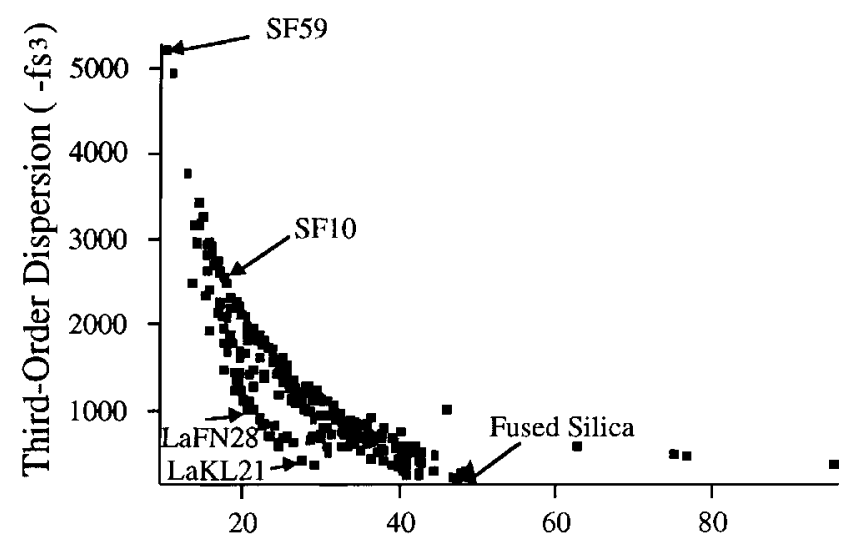

Prism Separation (cm)

Fig. 4. TOD vs prism separation in the oscillator for various prism materials (Schott Glass)

terial is essential for good TOD compensation, by plotting the value of the residual TOD in a cavity containing a $4 \mathrm{~mm}$ long Ti:sapphire crystal, for various glass types listed in the Schott glass catalog [52]. The prism separation is chosen for near-zero GVD. Typically, high-dispersion glasses have high TOD and therefore longer limiting pulsewidths. This is consistent with a simple limitation: the laser will operate with a bandwidth over which the net group delay in the laser is constant to within a fixed allowable error (experimentally determined to be $\sim 2 \mathrm{fs}$ ). If TOD is the limiting dispersion error, then the error is proportional to $\phi^{\prime \prime \prime}\left(\omega_{0}\right) \Delta \omega^{2}$. The allowable bandwidth (squared) $\Delta \omega^{2}$ thus scales inversely with TOD. Experimentally and theoretically, the pulse duration in these lasers has always proven to be compressible to the bandwidth limit, although the pulse in general emerges from the laser with some chirp due to optical path length in the Ti:sapphire crystal and the output coupler.

\section{Fundamental limits of lasers}

In order to generate even shorter (sub-10 fs) pulses from solid-state lasers, the next higher-order dispersion, third-order dispersion (TOD), must be eliminated. A very simple method to overcome this limit is to operate the Ti:sapphire laser at a center wavelength of $850 \mathrm{~nm}$, where, by using a fused silica prism pair and a short $2 \mathrm{~mm}$ Ti:sapphire crystal, simultaneous near-zero second- and third-order dispersion can be obtained. We used such a laser design to generate the first sub-10 fs pulses directly from a laser [5] and to explore the fundamental limits of pulse duration in modelocked lasers. In addition to operating the laser near $850 \mathrm{~nm}$ to eliminate TOD, we also replaced the dielectric mirrors with metallic silver reflectors, to eliminate any limitations due to the finite reflectivity bandwidth of dielectric mirrors. We found that the shortest pulse we could obtain from this laser was 7.5-8.5 fs [5], measured by using conventional autocorrelation, even though we could obtain very broad bandwidths of $\geq 170 \mathrm{~nm}$ FWHM.

To determine why the pulsewidth from the Ti:sapphire laser was limited to $\sim 8 \mathrm{fs}$, even though very broad bandwidths (with FWHM to support a 4 fs pulse) could be generated, we developed self-consistent numerical models of the laser, taking into account the interplay between the exact intracavity dispersion, gain lineshape, Kerr lensing, and selfphase modulation [7,42-44]. The total dispersive phase delay assumed in our models includes the dispersion of the crystal and the prism pair, as well as the resonant dispersion of the laser transition. In some cases we included a finite response time for the Kerr nonlinearity to test if such an effect could limit the modelocked bandwidth. In more-challenging simulations we included spatial effects, including diffraction and self-focusing of the intracavity pulses. The Kerr-lens modelocked laser is unique in that there is an intimate coupling of the spatial and the temporal evolution of the pulse. This uniqueness makes it challenging to develop a complete understanding of the operation of this laser, although the basic concept of Kerr-lens modelocking was published over two decades ago [53]. A complete model of these lasers must include the evolution of the optical pulses in space and time, particularly for the sub-20 fs regime of operation. We developed the first space-time coupled model of a modelocked laser, which simulates the propagation of a pulse as it bounces back and forth between the laser end-mirrors.

Several approaches have been used in the past to model the behavior of ultrashort-pulse Ti:sapphire lasers. Analytic theories treat the steady-state pulse propagation by using an extended ABCD formalism [53] and/or using a "master equation" approach [50]. These one-dimensional numerical models concentrate on the multipass dynamics of the pulse as it evolves toward steady state, taking into account the balance between self-phase modulation in the laser crystal, and group-delay dispersion due to the prisms (or chirped mirrors). Thus, the output pulse shape and duration are determined by a soliton-shaping mechanism, similar to pulse propagation in optical fibers [55]. This approach had been used for many years to model the behavior of dye lasers [56] where saturation effects cause pulse shortening down to the limit where soliton-like effects begin to play an essential role. However, it was subsequently demonstrated that even in the case of dye lasers [57], the soliton shaping mechanism works only in the atypical case of weak amplitude shaping and long pulses.

Our simulations show that a stable steady-state pulse is obtained primarily by a balance of self-phase modulation by the net negative dispersion of the intra-cavity components, similar to a soliton process. However, the Ti:sapphire laser can operate in a much richer parameter space than that predicted by analytical soliton theory. For example, Fig. 5a shows two experimentally measured output spectra from a laser, where the only parameter changed in the laser to obtain these two spectra is the insertion of one prism, changing the net intracavity dispersion. Figure $5 \mathrm{~b}$ shows the results of numerical simulations of the laser, discussed in more detail below. The laser can operate continuously, as the spectrum is adjusted continuously from the single-peak spectrum to the double-peak spectrum. The pulsewidth and shape also change continuously as the spectrum is altered. (Experimental results on pulseshape measurements will be discussed below.) Thus the Ti:sapphire laser, when operating in an ultrabroad-bandwidth mode, can generate a variety of stable pulseshapes.

The behavior of the laser can be understood in a simple way by considering the dispersion in the cavity. Figure 6 plots the calculated dispersion in the cavity corresponding to the two curves of Fig. 5. The dispersion is primarily 4OD- 

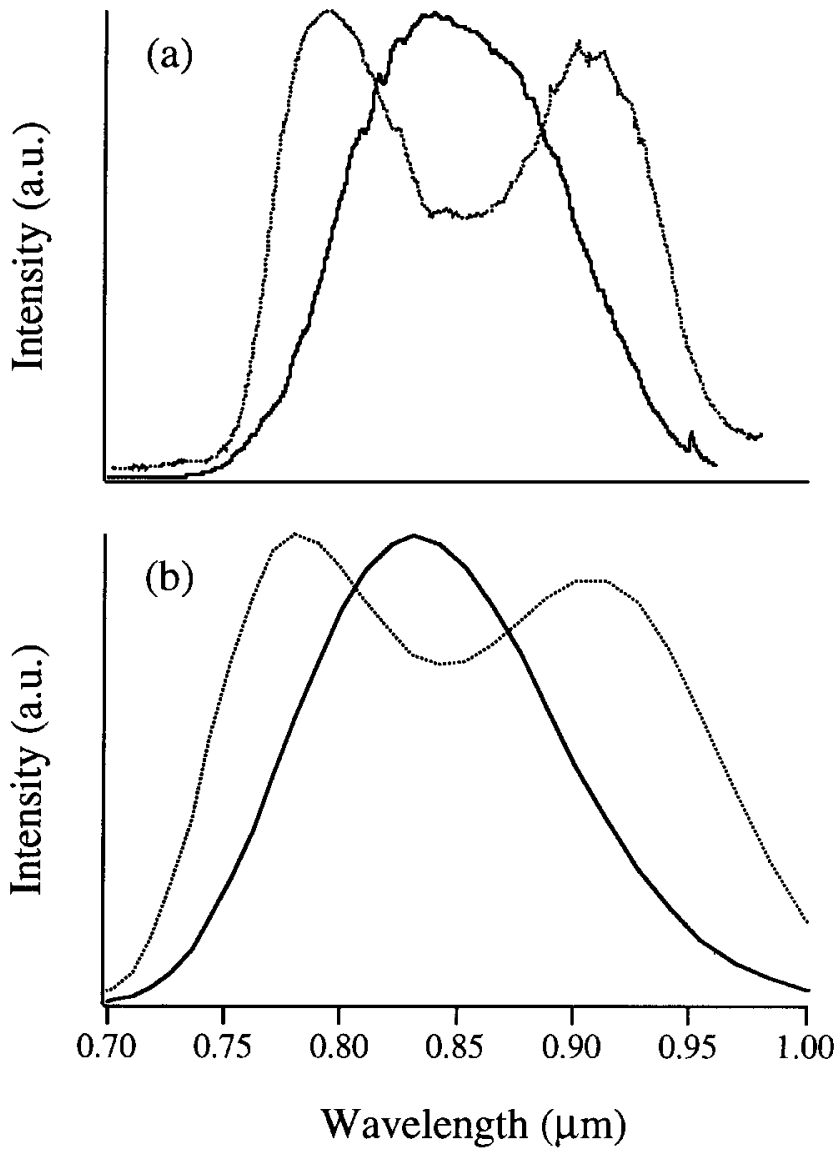

Fig. 5a,b. Power spectrum out of a Ti:sapphire oscillator: a experimental; b theoretical, for two values of dispersion in the laser

limited, since the shape of the dispersion curve is cubic. The shape of the spectrum corresponds to the shape of the dispersion curve. A heuristic picture of spectral dynamics is illustrated in Fig. 7. Parts of the spectrum which have a relatively short group delay will move towards the leading edge of the pulse, which will experience a red-shift due to selfphase modulation (which results from the same nonlinear index which causes self-focusing). The reverse happens for the parts of the spectrum that move to the back of the pulse. The overall negative dispersion at the extreme short and long wavelengths thus acts to "confine" the spectrum, and keep it from broadening indefinitely. However, in this case, for wavelengths near $850 \mathrm{~nm}$, where there is positive dispersion, the spectrum will spread, resulting in a "dip". The width of the spectrum is determined primarily by the wavelength region for which the group delay is constant within $\sim 2 \mathrm{fs}$, corresponding to a phase shift of approximately one wave. In this picture, it also becomes clear that higher-order dispersion in such a laser can be beneficial, allowing one to broaden the wavelength range over which the dispersion is small, while still using negative dispersion to confine the spectrum. In fact, for some values of combined fourth-order and negative GVD the output pulses have close to a sech ${ }^{4}$ time dependence [58, 59 ], instead of the usual $\operatorname{sech}^{2}$ or Gaussian shapes (which are usually assumed). A sech ${ }^{4}$ time dependence is in general desirable, since it corresponds to a sharper rising-edge than conventional $\operatorname{sech}^{2}$ pulses. The fact that such strongly modulated pulses are extremely stable in the Ti:sapphire laser

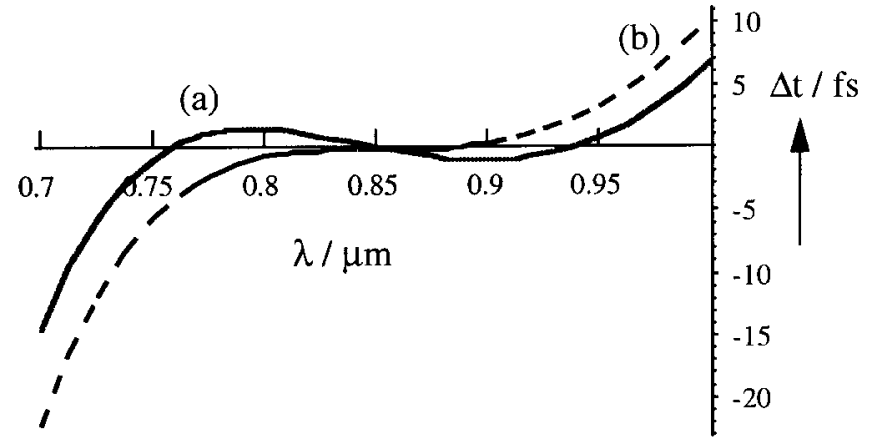

Fig. 6. Group delay vs wavelength for the two spectra in Fig. 5. Curve (a) corresponds to the double peaked spectrum, while (b) corresponds to the single peaked spectrum

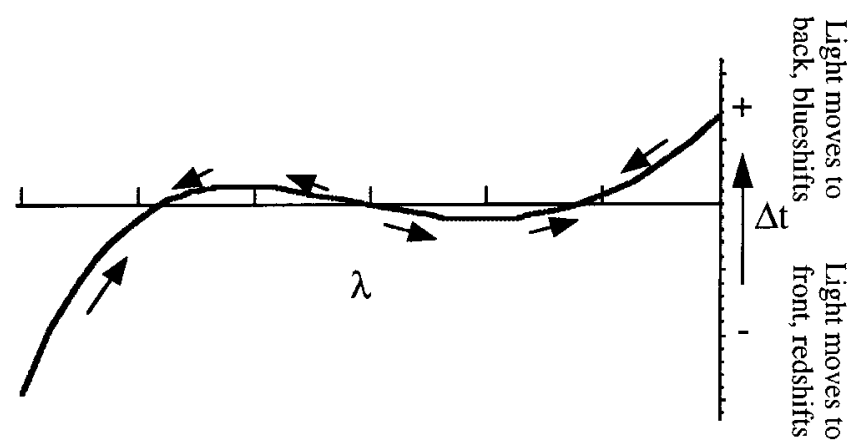

Fig. 7. Heuristic picture of the effect of self-phase modulation in the cavity. Dispersion combined with self-phase modulation causes colors with negative group delay to red-shift, while those with positive group delay blue shift. The spectral energy thus concentrates at two wavelengths, as in Fig. 5

indicates that a simple soliton picture or analytical masterequation approach to pulse evolution in these lasers is of limited applicability and can lead to incorrect conclusions. For the limiting case of zero second-order dispersion, the soliton theory predicts a collapse of the soliton; however, in practice stable pulses can propagate under such conditions, but their shapes differ significantly from a sech ${ }^{2}$ profile. We will see below that this situation becomes even more pronounced when spatial effects are taken into account.

For very broad-bandwidth pulses, it is not possible to describe the group delay in terms of a Taylor series expansion, since the dispersion curves are not simply quadratic or cubic, and since the various higher-order terms may not be a rapidlydecreasing function. Therefore, for accurate modeling of the cavity, the net group delay must be used, and not individual higher-order dispersion terms. Such an approach is straightforward to implement using numerical models [7, 42-44]. Results of this dispersion model of the laser are shown in Fig. 5b, and the excellent agreement between the model and data demonstrates that the model includes the most significant pulse-shaping effects in the laser. Kerr lens modulation in this case is modeled simply by attenuating all light that falls below a threshold intensity of $1 \%$ of the peak intensity, and the results are insensitive to the exact form of this amplitude modulation. Altering the dispersion characteristics in the model allows us to predict with confidence that the ultimate limits to pulse duration in a Kerr-lens modelocked laser are at least as short as $5 \mathrm{fs}$. This value is obtained by using zero GVD and 4OD compensation similar to that shown in 
Fig. $6 \mathrm{~b}$, but with a magnitude 10 times smaller. The use of 4OD results in shorter stable pulse durations than in the case of simple negative GVD compensation. This result may seem paradoxical if a soliton picture of pulse formation is taken too literally - in soliton theory, GVD is necessary for pulse formation and higher-order dispersion is undesirable. However, in light of the more complete modeling discussed herein, and the simple argument illustrated in Fig. 7, it is completely reasonable to expect that a dispersion curve that flattens-out near the center but is negative at the edges will allow the pulse spectrum to broaden farther while still remaining well confined.

\section{Space-time focusing in ultrashort-pulse lasers}

As discussed above, modeling of solid-state femtosecond lasers has advanced to such a point that we can now selfconsistently model the laser to obtain theoretical bandwidths and pulsewidths in excellent agreement with experiments [5, $7,10,42-44]$. However, physical interpretation of these numerical models is important to understand Kerr-lens modelocked lasers in detail. One of the most important features that distinguishes Kerr-lens modelocked lasers from other ultrafast modelocked lasers is the role played by spatial effects, including diffraction and self-focusing of the intracavity pulses. A complete model of these lasers must therefore include the evolution of the optical pulses in both space and time. This is particularly true for the sub-20 fs regime of operation. In addition, the astigmatic angles of incidence of the beam on the laser mirrors, together with the Brewster angle of the laser crystal, makes the problem inherently three dimensional. Finally, the large spectral bandwidth and dis-
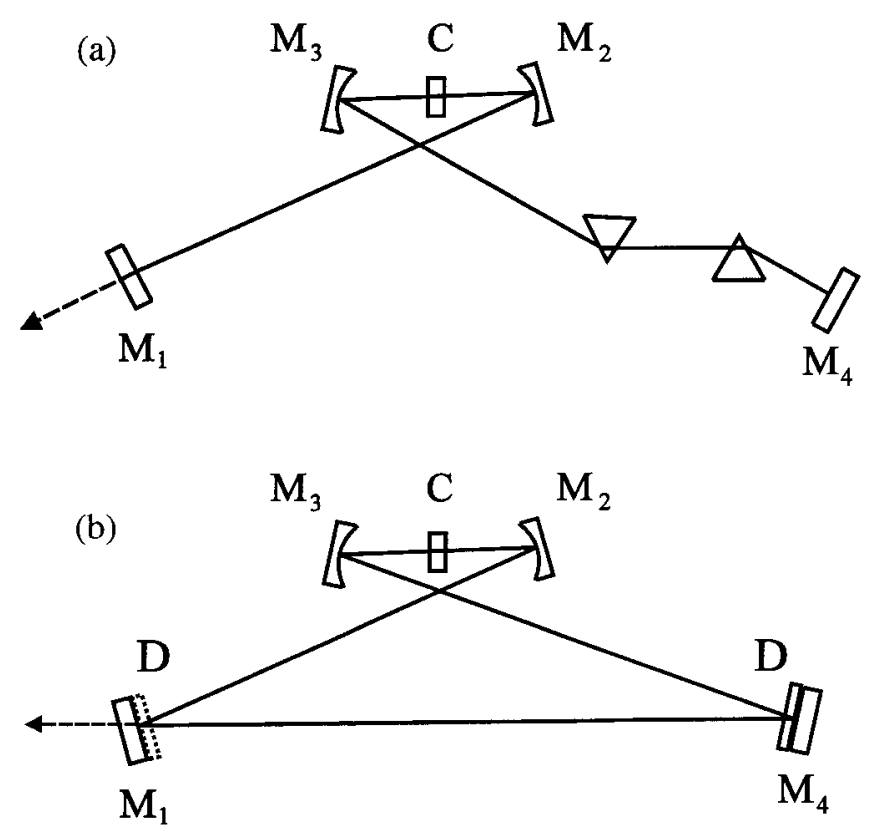

Fig. 8. Schematic of the linear and ring resonators used in our model calculations. The focal length of the curved mirrors is $3.5 \mathrm{~cm}$, the crystal length is $0.23 \mathrm{~cm}$, and D is the dispersion control. The distances for the linear resonator are M1-M2 $=58.8 \mathrm{~cm}, \mathrm{M} 2-\mathrm{C}=3.443 \mathrm{~cm}, \mathrm{C}-\mathrm{M} 3=3.438 \mathrm{~cm}$, and $\mathrm{M} 3-\mathrm{M} 4=101 \mathrm{~cm}$. For the ring resonator: $\mathrm{M} 1-\mathrm{M} 2=50 \mathrm{~cm}, \mathrm{M} 2-\mathrm{C}=$ $3.438 \mathrm{~cm}, \mathrm{C}-\mathrm{M} 3=3.449 \mathrm{~cm}, \mathrm{M} 3-\mathrm{M} 4=50 \mathrm{~cm}$, and M4-M1 $=64 \mathrm{~cm}$ persion within the laser means that the pulse duration varies dramatically depending on both the position and the direction of propagation of the pulse within the cavity [5]. Thus, even though the laser cavity is physically a linear rather than a ring cavity, modeling of the laser mode must assume an effective ring cavity to account for the asymmetry of the nonlinear self-focusing.

In two recent papers $[7,44]$ we introduced more complete numerical models where the evolution of the pulse in the laser cavity is described in three dimensions. Axial symmetry of the laser beam is assumed, which allows us to transform the frequency-dependent diffraction from two to three dimensions. We can thus observe, for the first time, the spatial evolution of the laser pulse from the initial stage to steadystate value, without making any assumptions about the nature of the nonlinear amplitude modulation. In these models, mode-locking is favored over cw operation simply because the restricted pump area in the laser crystal can result in higher net gain for a self-focused pulse than for low-intensity light. Alternatively, we can model a "hard aperture" in the laser. In our modeling, we consider two different resonator configurations: linear and ring, as shown in Fig. 8. To reproduce our experimental results, we assume that the dispersion control in the linear cavity is accomplished by a prism pair, while in the ring cavity, we concentrate the dispersion close to one or two flat mirrors to simulate a dispersion-controlled mirror compensated cavity [46]. Free-space pulse propagation in the region between the optical components is accomplished using Fresnel integrals, while the nonlinear propagation through the laser crystal is simulated by a split-step approach.

Figure 9 shows the evolution of the pulse spectrum and pulse duration at the output couplers of the linear and ring cavities shown in Fig. 8. After a transient oscillatory phase, the pulses evolve toward steady state, with small residual oscillations that depend on the precise tuning of the resonators. The important point to note here is that the final pulse spectra and pulse duration at the output coupler do not correspond to transform-limited pulses, but rather are positively chirped. The position of the transform limited pulse (time waist) occurs within the laser crystal. This can clearly be seen in Fig. 10, which shows the space-time focusing exhibited by the pulse in the crystal in steady state. This behavior has already been demonstrated experimentally in our previous work [5]. Thus, we conclude that the nonlinear Kerr-lens modelocked resonator tends to compensate for the "astigmatism" between the space and time foci inside cavity, and to match the beam waist in the crystal with the "time waist" of the pulse to maximize the self focusing. To show that the combined space-time focusing in the laser crystal greatly improves the selective properties of the nonlinear resonator, in Fig. 11 we plot the transmission of the resonator for a pulse that exhibits space-time focusing, as compared to one with no time focusing. We see that spacetime focusing ensures better transmission and better stability against intensity fluctuations (narrower peak). Thus, owing to the fast response time of the Kerr nonlinearity, spacetime focusing in Kerr-lens modelocked lasers is the main mechanism that sustains stable mode-locking. The role of dispersion compensation is to ensure that space and time foci are appropriately positioned to maximize the nonlinearity. 

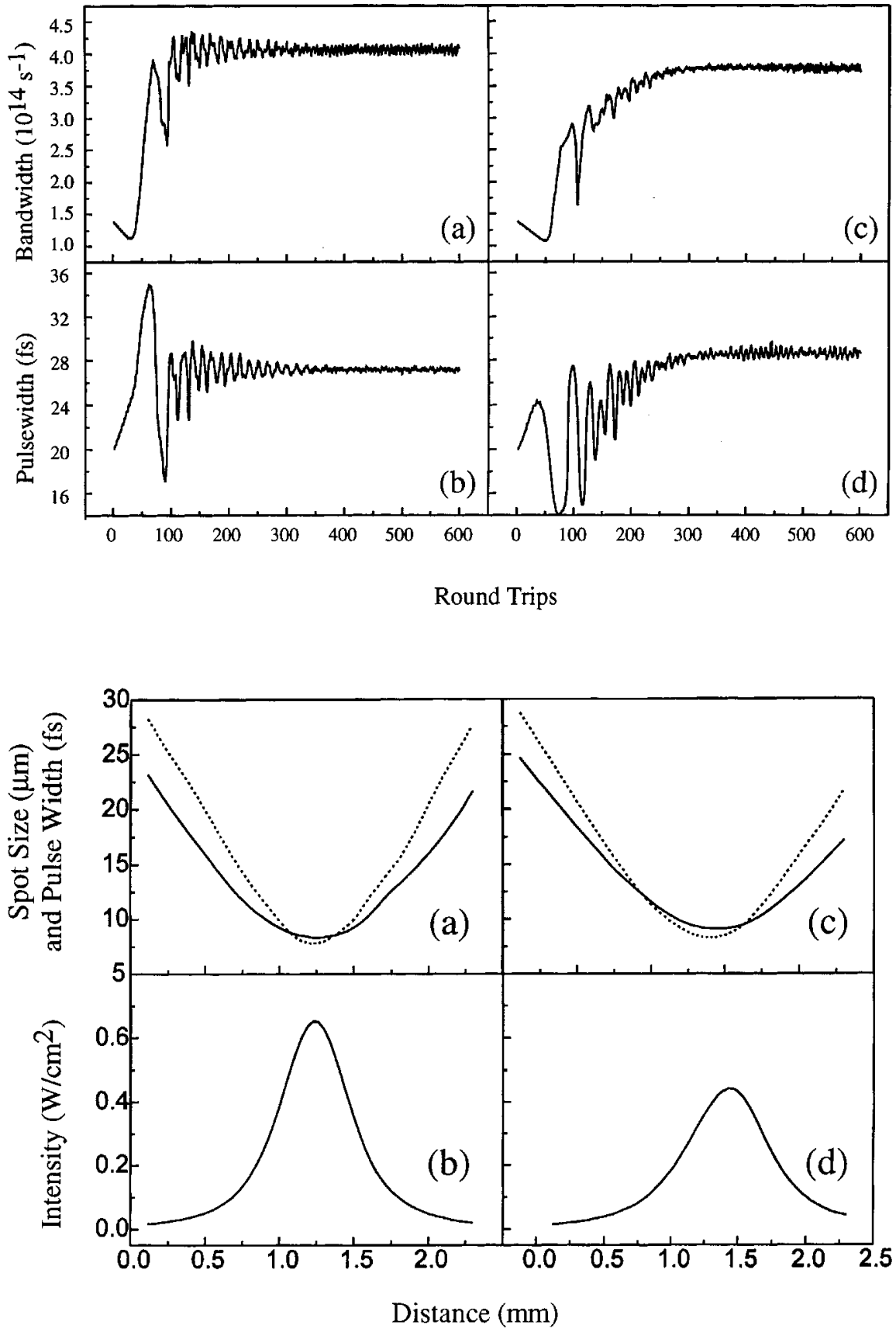

Fig. 9a-d. Evolution of the pulse bandwidth (a), (c), and pulse duration (b), (d) at the output coupler M1 vs the number of round trips for a linear resonator (a), (b), and for a ring resonator $(\mathbf{c})$, (d). The pulse duration at the output coupler is not transform limited

Fig. 10a-d. Space-time focusing in the laser crystal in steady state for a linear resonator (a), and for a ring resonator (c). Curves (b) and (d) show the peak intensity of the pulses versus distance in the crystal. Solid line - pulse duration; dashed line - spot size

The fundamental reason for this behavior is that the net intracavity dispersion is not a single entity: the positive dispersion within the crystal plays its own role in the pulse dynamics. Owing to the time focus in the crystal, and depending on the exact crystal thickness, the pulse that enters the crystal will be chirped. Self-focusing causes the crystal to act as a nonlinear spectral filter because the higher and lower frequencies that are positioned at the leading and trailing edges of the pulse exhibit weaker self-phase modulation than the peak of the pulse. Therefore, use of a thinner crystal will ensure broader modelocked spectra and shorter pulse durations than in the case of a thicker crystal, given equal dispersion compensation in the cavity. These considerations apply for both linear and ring cavities.

\section{Ultrashort pulse amplifiers}

The broadband, ultrashort-pulse, laser oscillators described in the first part of this paper are ideal sources of low energyseed pulses for high-power, ultrafast amplifiers. While it is possible to routinely generate pulses as short as $10 \mathrm{fs}$ pulses directly from a laser oscillator, for high-power amplifiers of terawatt peak powers, the pulse durations that can be generated from state-of-the-art systems at the present time are approximately $15-20 \mathrm{fs}[13-16,23,24,60,61]$. Typically, several design choices must be made in developing high-power ultrafast lasers, involving trade-offs between pulse energy, pulse duration, spatial quality, pulse contrast, pulse repetition rate, and wavelength. Nevertheless most systems have many features in common: a front-end laser, a pulse stretcher, sev- 


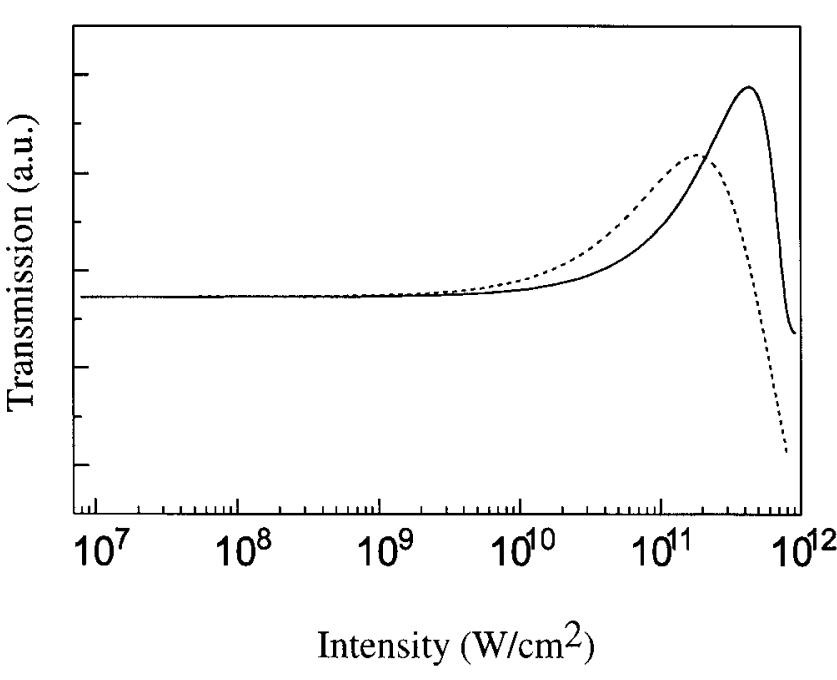

Fig. 11. Transmission of the resonator for optimally chirped pulse (solid line) and for an unchirped pulse (dashed line) as a function of peak intensity in the laser crystal

eral stages of amplification, followed by a pulse compressor. This method of stretching the pulse in time prior to amplification, in order to maintain fluences well below damage threshold in the optical materials in the amplifier, is known as chirped-pulse amplification (CPA) [12]. Following amplification, the pulse can be recompressed to a short pulsewidth by reversing the chirp process.

In designing amplifier systems for high-power pulses of duration $\ll 100 \mathrm{fs}$, there are two major effects that may limit the final pulse duration. First, the finite bandwidth of the gain medium results in narrowing of the pulse spectrum during amplification. Ti:sapphire is the broadest-bandwidth laser material known (FWHM gain bandwidth of $230 \mathrm{~nm}$ ); however, after amplification by factors of $10^{7}-10^{11}$, gain narrow-

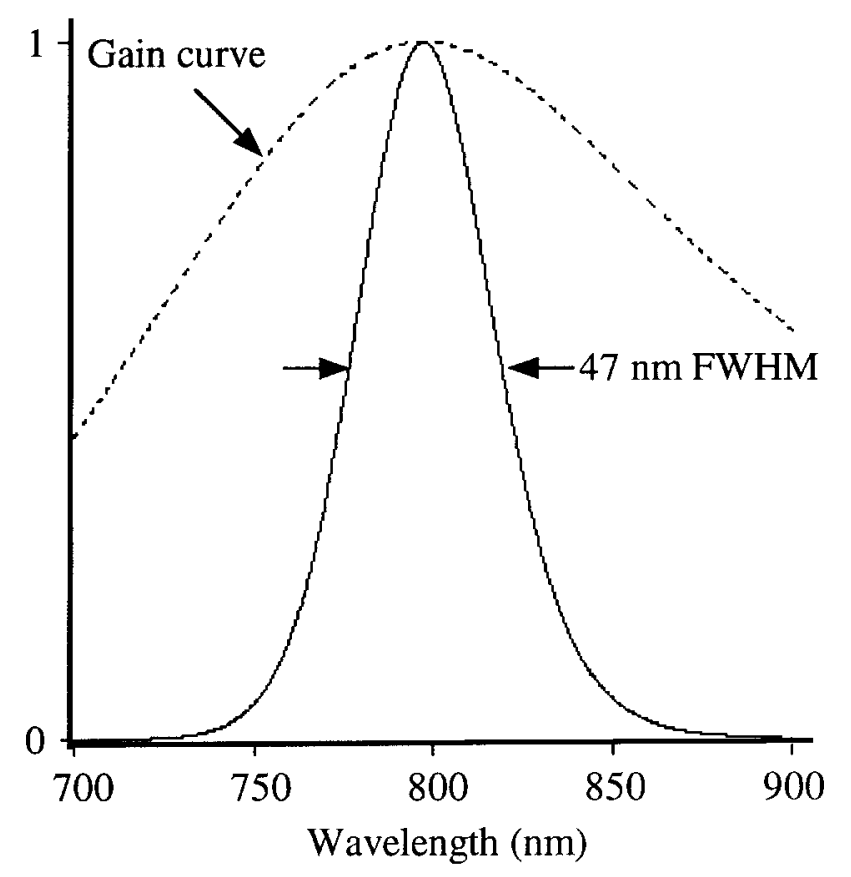

Fig. 12. Gain narrowing for an infinitely broad input spectrum at $10^{7}$ gain ing limits the output bandwidth. For the case of infinitely broadband input pulses injected into a Ti:sapphire amplifier with a gain of $10^{7}$, the amplified output spectrum is $\approx 47 \mathrm{~nm}$ FWHM, as shown in Fig. 12. This bandwidth is capable of supporting pulses as short as $18 \mathrm{fs}$, at millijoule pulse energies. For the highest energy (TW) output pulses, the gain-narrowing limit is $\approx 25$ fs for Ti:sapphire. For all other amplifier materials, the gain-narrowing limit is even more severe. In practice, it is not necessary to have infinitely broad bandwidth input pulses in order to obtain amplified output spectra as broad as $47 \mathrm{~nm}$. This is because the input pulse spectrum from the oscillator can be adjusted to compensate for gain narrowing and for the related effect of red shifting of the spectrum during amplification. This is illustrated in Fig. 13. Red shifting of the pulse spectrum results from the fact that the leading edge of the chirped pulse being amplified (longer wavelengths) sees a higher gain than the trailing edge. It is therefore not desirable to center the wavelength of the low-energy seed pulses at $800 \mathrm{~nm}$, since then the red shift and gain narrowing causes the pulse spectrum to severely narrow and shift from the peak of the gain of the amplifier. In contrast, broad amplified bandwidths at high energies can be obtained by positioning the input spectrum on the short wavelength side of the desired output, as shown in Fig. 13. It is interesting to note that the spectral output from 4OD-limited prism-dispersion controlled Ti:sapphire lasers is ideal for further amplification, and that in the case of gainnarrowing limited amplification, the use of shorter-duration seed pulses will not alter the resulting output. The effects of gain-narrowing can be ameliorated to some extent by introducing compensating spectral attenuation to flatten the net gain profile in the laser amplifier [62-64]. However, this gain reshaping also tends to result in phase distortion (due to the gain-modulating elements) and reduced pulse spectral content far away from the center wavelengths. The net result is a modest reduction (10-20\%) in pulse duration at the expense of temporal "cleanliness" of the pulse. It is of interest to note that shaping the spectrum of the input pulse as we have done does not inherently compromise the fidelity of the amplified pulse. As mentioned previously, the output from the mode-locked Ti:sapphire laser is essentially transform-limited, even in the case of a spectrum that deviates significantly from the $\mathrm{sech}^{2}$ shape. In the case of perfect dispersion compensation in the CPA system, the output pulse shape is thus simply the transform limit of the output spectrum.

The second limiting effect on pulse duration in CPA systems is higher-order dispersion. The CPA technique requires a very accurate chirp and recompression of an ultrashort laser pulse. Since typical stretching factors are in the range of $10^{4}$, with such large stretch factors it is quite easy to introduce unwanted higher-order dispersion into the optical system. For pulse durations $>30 \mathrm{fs}$, both GVD and TOD must be compensated. This compensation may be achieved by adjusting the grating separation and incident angle in either the pulse stretcher or compressor. For sub-30 fs pulses, yet another degree of freedom is required to minimize the residual fourth-order dispersion in the system. Given that the contribution of most optical materials to 4OD is negative, material can be added to the beam path to cancel 4OD. Alternatively, for constant GVD, the magnitude of the TOD and $4 \mathrm{OD}$ increases as the grating groove density is increased. 


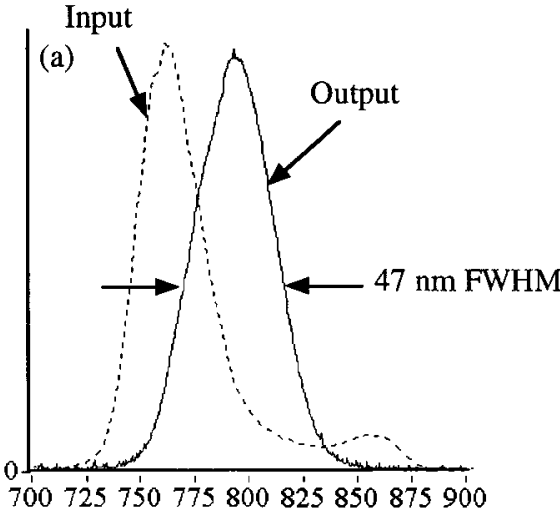

Wavelength (nm)

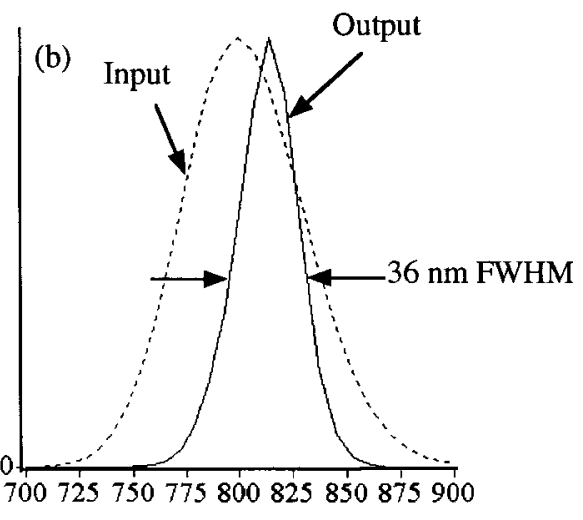

Wavelength (nm)
Fig. 13a,b. Gain narrowing for an optimally shaped input spectrum (a), and for a non-optimally shaped one (b) . The spectrally-averaged gain in each case is $7 \times 10^{7}$
In previous work, we found that for the typical amount of material present in the beam path of a multipass amplifier system, the residual 4OD was minimized by using 600 line/mm gratings. We also found that by using an additional prism-pair in the amplifier [15,24], we could eliminate all higher-order dispersion up to and including fourth-order, over extremely large bandwidths, to produce high-quality, $20 \mathrm{fs}$ pulse durations. This will be discussed below. Other possibilities for compensating higher-order dispersion are to use different groove densities for the stretcher and compressor (although this choice is somewhat system-specific) or to use specially-designed lenses to compensate for 4OD [65,66]. The most desirable and flexible method to eliminate highorder dispersion would be to introduce active control over the spectral phase in a spatially and spectrally dispersed region of the amplifier system [67-69]. However, at present, such pulse-shaping systems are somewhat difficult to use and are lossy.

Another factor that effects pulse shape is the overall spectral bandpass of the system. The finite size of the pulse stretcher and compressor gratings place hard cutoffs on the spectrum. For good pulse fidelity, it is important that the spectral bandpass be $\sim 3$ times the spectrum FWHM. Even in this case, spectral clipping will result in a background of uncompressed light. This effect, and the finite bandwidth over which dispersion is well compensated, are generally the limiting factors in intensity contrast from these highenergy ultrafast systems. The use of low-groove density gratings makes it more practical to obtain a large spectral bandpass.

\section{Specific amplifier systems}

In our work, we developed two novel ultrashort-pulse amplifier systems $[13-15,23,24]$. The first is a high-repetitionrate laser system, which generates several millijoules of energy at $\mathrm{kHz}$ repetition rates. The second system is a high peak-power laser system, capable of generating approximately $3 \mathrm{TW}$ of peak power at $10 \mathrm{~Hz}$ repetition rates (Fig. 14). Our amplifier design philosophy has been to reduce the complexity as far as possible, while optimizing the systems for ultrashort-pulse operation. We use highly-doped Ti:sapphire crystals in the amplifier, to reduce uncompensatable higherorder dispersion in the system, together with all-reflective, low-aberration, stretcher, and compressor designs. We also use relatively modest stretch factors (600-1200 g/mm gratings), which stretch the pulses from 12 fs to $\approx 50-80 \mathrm{ps}$. This allows us to compress the output pulses back to near their original pulse duration following amplification.

For our most recent work, we developed a $1 \mathrm{kHz}$ repetitionrate, Ti:sapphire-based, amplifier system, which can simultaneously generate high peak and average powers of $0.2 \mathrm{TW}$ and $4 \mathrm{~W}$, respectively [23,24]. The laser system generates $4 \mathrm{~mJ}$ energy pulses, with $20 \mathrm{fs}$ pulse duration, and is shown in Fig. 15. The seed pulses are derived from a self-modelocked Ti:sapphire oscillator [3], with the spectral shape and peak wavelength $(760 \mathrm{~nm})$ adjusted to compensate for spectral shifting and shaping in the amplifier. This allows us to maximize the amplified spectral bandwidth. The short pulses are then stretched in time to a duration of $50 \mathrm{ps}$, using an all reflective pulse stretcher [13]. Higher-order dispersion com-

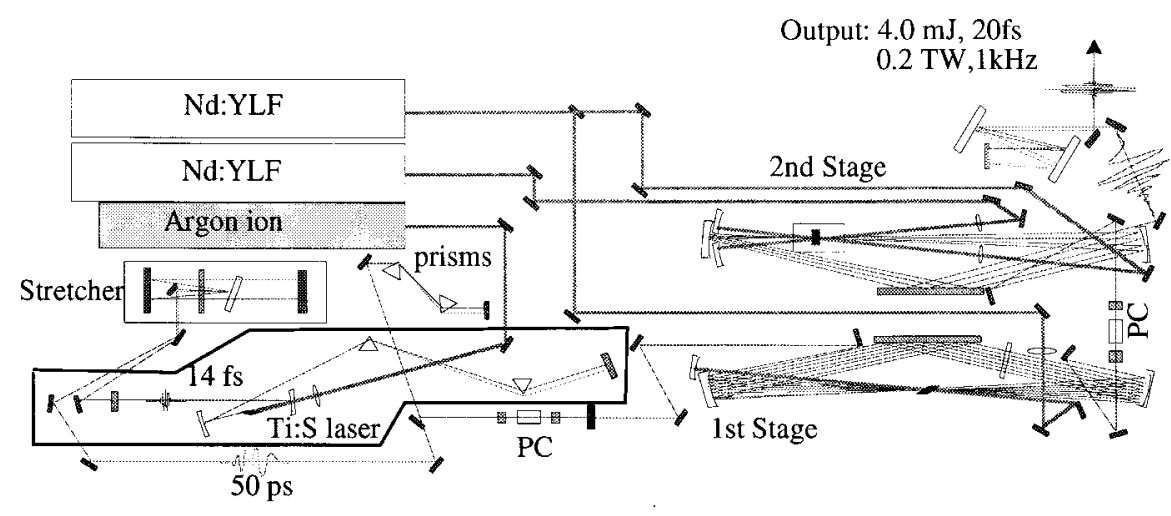

Fig. 14. Schematic diagram of a $0.2 \mathrm{TW}, 1 \mathrm{kHz}$, Ti:sapphire CPA system 


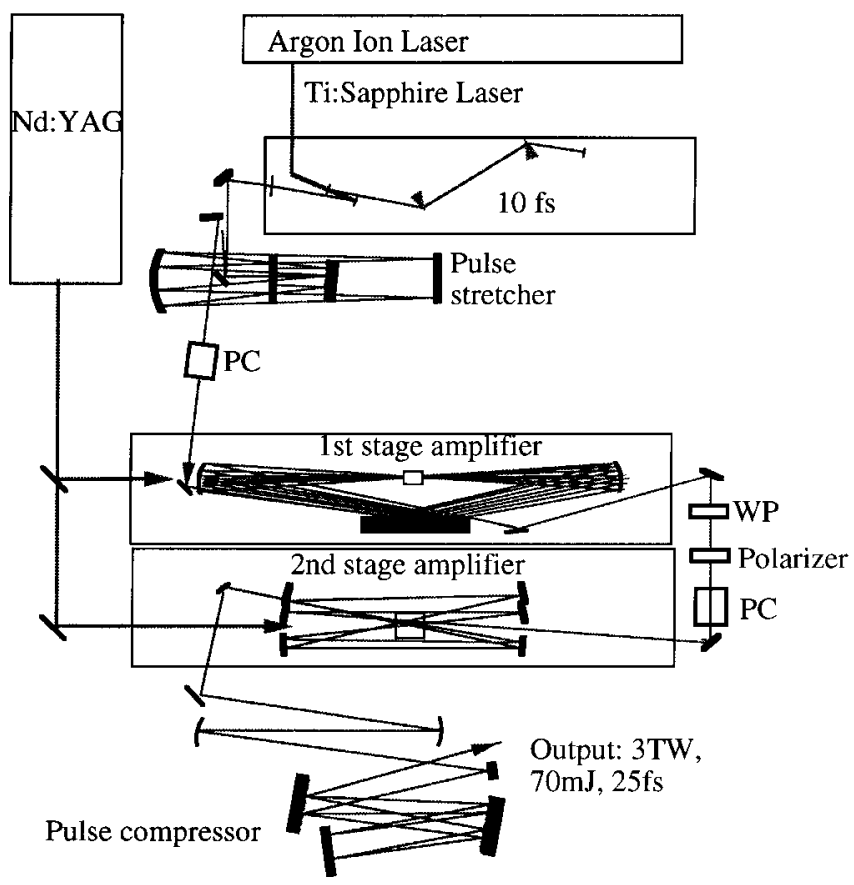

Fig. 15. Schematic diagram of a $3 \mathrm{TW}, 10 \mathrm{~Hz}$, Ti:sapphire CPA system

pensation is achieved by propagating the beam through an SF18 glass prism pair. Optimization of the stretcher grating angle and position, and the prism separation, allows us to compensate dispersion in the system up to fourth order [70].

The laser system consists of two stages of amplification, both using multi-pass ring designs, which reduce the amount of material in the amplifier compared with regenerative amplifier designs. The ring design is very flexible, since the crossing point can be moved to an arbitrary point in the ring to optimize the mode size in the amplifier crystal. One of the major problems with high average power ultrafast systems is thermal lensing induced by the heat load of the large pump laser fluence on the amplifier crystal. Thermal lensing can lead to a mismatch between the pump and laser mode in subsequent passes through the laser amplifier, and to possible optical damage. In the case of the first pre-amplifier stage, the beam is approximately at a waist when passing through the crystal, which minimizes the influence of lensing on the beam divergence. However, for the second power amplifier stage, this is not the case. The beam propagation can be severely influenced by thermal lensing. Although the thermal lens can be compensated for to some extent using corrective optics (i.e., lenses), high-order aberrations on the pulse can be introduced that are difficult to correct.

Given our goal of obtaining the highest possible focusable intensity, we chose to reduce, rather than compensate for, the thermal lens. Ti:sapphire has excellent thermal characteristics even at room temperature, where the thermal conductivity is $46 \mathrm{~W} / \mathrm{m} \mathrm{K}$. However, at $77 \mathrm{~K}$, the conductivity jumps to over $2000 \mathrm{~W} / \mathrm{m} \mathrm{K}$. The reduced thermal gradients result in reduced lensing, with the focal length of the lens being directly proportional to the thermal conductivity [71]. The thermal lens is also inversely proportional to the change of refractive index as a function of temperature, $\mathrm{d} n / \mathrm{d} T$, which is also more favorable at low temperature. As a result, by cooling the Ti:sapphire crystal, it is possible to reduce this effect from $13 \times 10^{-6} / \mathrm{K}$ at $300 \mathrm{~K}$, to $3 \times 10^{-6} / \mathrm{K}$ at $125 \mathrm{~K}$ [72]. In our case, this corresponds to improving the focal length of the thermal lens, in the case of $22 \mathrm{~W}$ absorbed pump power, from $f_{\text {therm }}=45 \mathrm{~cm}$ at $300 \mathrm{~K}$, to $f_{\text {therm }}=5.6 \mathrm{~m}$ at $125 \mathrm{~K}$. We used a simple, liquid nitrogen-cooled cryostat to accomplish this. The first amplifier stage was pumped with $8.5 \mathrm{~W}$ from a Quantronix 527 Nd:YLF laser [23]. The second stage uses identical 1 meter ROC mirrors as the first stage, with the pump spot size increased from $\sim 500$ to $\sim 1 \mathrm{~mm}$. The pump power was $26 \mathrm{~W}$, and it was injected with seed pulses of energy $700 \mu \mathrm{J}$ from the first stage. Given the output energy of $7 \mathrm{~mJ}(7 \mathrm{~W})$, this corresponds to a second-stage efficiency of $\approx 30 \%$. After compression using a pair of $600 \mathrm{~g} / \mathrm{mm}$ gratings, the output energy is $4 \mathrm{~mJ}$ for our compressor throughput of $57 \%$. The output beam focuses to near the diffraction limit, and the ASE background (measured by blocking the seed pulse) is $<3 \mu \mathrm{J} /$ pulse. In the near future, we plan to replace our compressor gratings with higher-efficiency versions, which should allow us to obtain $>5 \mathrm{~mJ}$ at $1 \mathrm{kHz}$.

The second amplifier system we developed is shown in Fig. 15 [13,14]. This system can generate pulses with a duration of $26 \mathrm{fs}$, and with an energy of $75 \mathrm{~mJ}$, from a simple two-stage Ti:sapphire amplifier system. The peak power of these pulses is thus $3 \mathrm{TW}$, and the repetition rate is $10 \mathrm{~Hz}$. The amplifier design consists of two multi-pass amplifiers and is relatively simple and compact. As in the case of the high-repetition rate system described above, we use an all-reflective, low-groove density, grating stretcher and compressor, combined with relatively short material path length. This design allows us to minimize higher-order dispersion in our amplifier. The result is a system which generates multiterrawatt, transform-limited pulses with good beam quality and low ASE levels, at a duration near the theoretical limit imposed by gain narrowing in Ti:sapphire.

\section{Precise measurement of ultrashort-pulses}

As a further confirmation of our understanding of the fundamental limiting physics of the Ti:sapphire laser, we developed sophisticated measurement techniques for 10-20 fs pulses $[10,11]$, in collaboration with Trebino and his group. In addition to its utility for understanding the fundamental physics of modelocked lasers, accurate pulse characterization is essential for many ultrashort-pulse applications. For example, in coherent X-ray generation via harmonic up-conversion [27, 34,73,74], laser-based particle acceleration $[32,75]$, or coherent control of wavepackets in ultrashort chemical reactions [76-78] the pulse shape can be tailored to optimize the results. Such precise shaping can be achieved only through proper understanding of the electric field of the pulse (amplitude and phase). Finally, in ultrashort-pulse (sub$30 \mathrm{fs}$ ) amplifier systems, as discussed above, accurate characterization of the amplitude and phase of the output pulses is key to perfecting the optical system.

Prior to about 1995, ultrashort pulse systems were typically characterized by measuring only the power spectrum and second-order autocorrelation. The former provides only spectral amplitude information, without spectral phase information, and therefore the Fourier transform gives information on the ultimate pulse duration that could be obtained if the frequencies were all in phase. This situation has led to many 
inaccuracies in the literature, particularly for sub- $30 \mathrm{fs}$ pulses, because of the significance of higher-order dispersion both in determining the pulse shape generated by the laser, and the dispersive propagation of these pulses. The transformlimited FWHM pulse duration (assuming flat spectral phase) of a pulse with a spectral FWHM bandwidth of $40 \mathrm{~nm}$ will be $18 \mathrm{fs}, 24 \mathrm{fs}$, or $45 \mathrm{fs}$, depending on whether a sech ${ }^{2}$, Gaussian, or flat-topped pulse shape is assumed. To complicate matters further, traditional autocorrelation techniques tend to underestimate the pulse duration, since the spectrum is almost always modulated or clipped due to finite bandwidths of mirrors and gratings, and thus the spectral and temporal shapes are never purely Gaussian or sech ${ }^{2}$. These limitations apply equally to conventional and interferometric autocorrelation, since the interferometric trace contains essentially the same data as the spectrum combined with the intensity autocorrelation $[79,80]$. However, the interferometric technique adds the complication of a high degree of sensitivity to alignment.

In this light, the recent development of new measurement techniques, which allow complete characterization of ultrashort pulses (amplitude and phase), are a particularly significant development in the field $[9,81]$. We have worked in collaboration with Trebino et al. to apply one of these measurement techniques, Frequency-Resolved Optical Gating (FROG), to ultrashort pulses $[10,11]$. In traditional autocorrelation techniques, the amplitude of a nonlinear signal is recorded as a function of the delay between two identical replicas of a short pulse. In FROG, both the spectrum and amplitude of the nonlinear signal are recorded as a function of delay. FROG applies well-developed phase-retrieval algorithms from spatial image analysis to solve $[82,83]$ the problem of retrieving a pulse shape from the measured data. It has the added benefit of having very strong constraints in the different fourier domains of the algorithm, which retrieves the pulse by a self-consistent iterative process. A great advantage of FROG is that it also has internal self-consistency checks on the data, which prevent systematic measurement errors. For example, if the SHG FROG trace is integrated over the time axis, the result, known as the frequency marginal of the trace, can be shown to be proportional to an autoconvolution of the fundamental power spectrum. This can be measured independent of the FROG algorithm, allowing one to detect problems with the experimental set-up. Such checks are completely lacking in traditional autocorrelation. FROG has also been shown to be very robust against noise.

FROG can be applied with nearly any optical nonlinearity, but we have focused on two versions in particular: Second Harmonic Generation (SHG) FROG, which is ideal for weak unamplified pulses, and Transient Grating (TG) FROG, which is perfectly phase matched and is ideal for high-power ultrashort pulses. SHG FROG is similar to traditional autocorrelation, except that the spectrum and amplitude of the second-harmonic signal is recorded as a function of delay between two pulses. In TG FROG, two identical beams are overlapped in a medium (e.g., fused silica) to generate an induced grating. A third portion of the pulse scatters off the induced grating from the first two beams, to produce a fourth beam at the same frequency, which is the nonlinear signal to be measured. In this section we will report on the application of these two FROG geometries to measure the shortest, fully-characterized pulses to date.
Experimentally, there are many difficulties involved in measuring ultrashort-pulses with bandwidths of $100 \mathrm{~nm}$ or greater $[10,11]$. The optical elements in the measurement apparatus cannot limit the bandwidth and the frequency response of any detector used must be accurately calibrated. Finally, the phase matching bandwidth of the frequency doubling medium must be maximized. This finite phasematching bandwidth becomes less of a problem as the crystal thickness is reduced, and the thinnest possible crystals are generally used for ultrashort-pulse applications (at the expense of signal level). Using very thin KDP crystals $(\approx 50 \mu \mathrm{m}$ we found that phase matching of our extremely large bandwidths $(\approx 170 \mathrm{~nm})$ was a problem, since the crystal has to be oriented very precisely to achieve optimum phasematching. This is an iterative process, and even then is not perfect for ultra-broadband pulses. More recently, we used a thin film $(0.4 \mu \mathrm{m})$ containing highly oriented NLO organic chromophores with a large nonlinearity as a nonlinear medium [84]. These films were made of tricyanovinylaniline (PhTCV), which was specifically developed for Ti:sapphire ultrashort-pulse SHG applications. A poled TCV was used in an SHG-FROG to characterize $13 \mathrm{fs}$ pulses from a modelocked Ti:sapphire oscillator. The retrieved electric field is shown in Fig. 16.

The ability of FROG to fully characterize ultrashort pulses has proved extremely useful in verifying the predictions of theoretical models of our laser. As discussed above, numerical models of the Ti:sapphire laser are necessary to describe the operation in the sub-20 fs regime, because analytical models can describe the operation only in a very limited regime. As discussed above, in order to generate broadbandwidth pulses with which to test our models, we operated at $850 \mathrm{~nm}$, where both the second- and the third-order dispersion in the laser cavity were near zero. Therefore, the femtosecond pulses were shaped and sustained by fourthorder dispersion. Fourth-order dispersion results in a large spectral region of near-zero group delay, which allows broader bandwidth operation than in the case of second-order dispersion. The output spectra from the laser were charac-

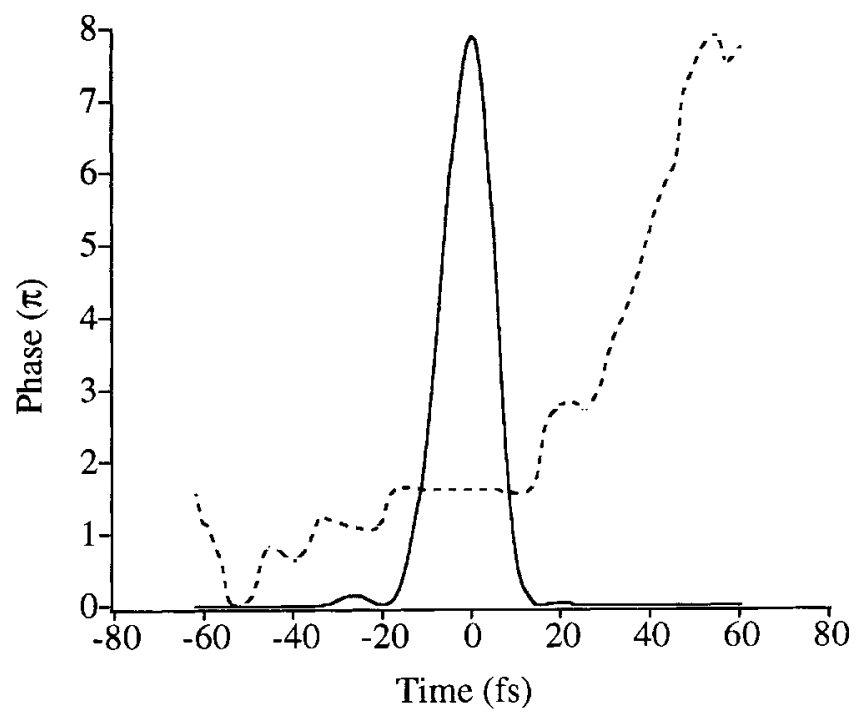

Fig. 16. Measured pulse temporal intensity and phase for a 13 fs pulse using SHG FROG and single layer non-linear SHG medium (TCV) 
FROG spectrograms of a 9-fs pulse

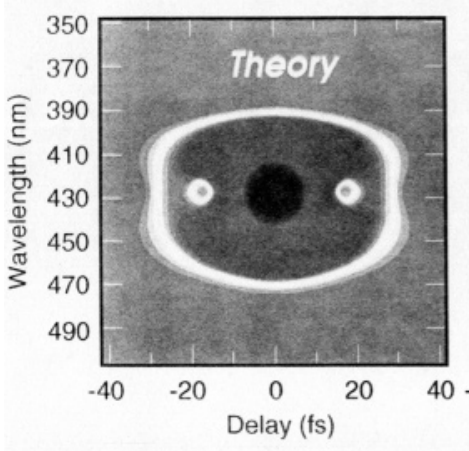

(a)

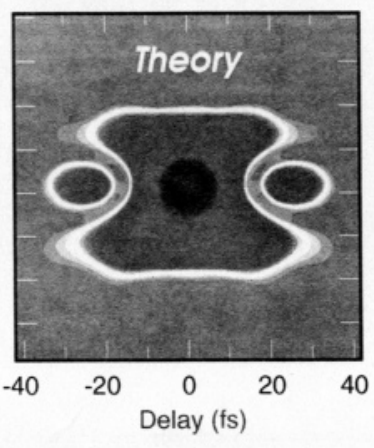

(b)

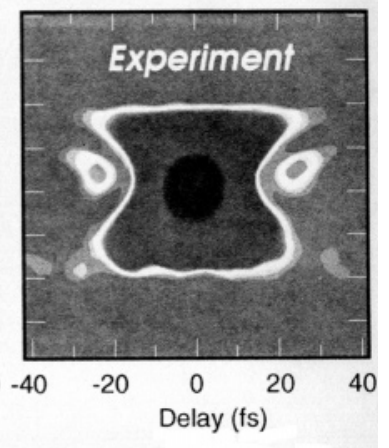

(c)
Fig. 17a-c. Comparison of the SHG-FROG trace for two theories of oscillator operation and of our experimentally measured pulse. The key differences are the two islands present in (b) and (c) which are not present in (a)

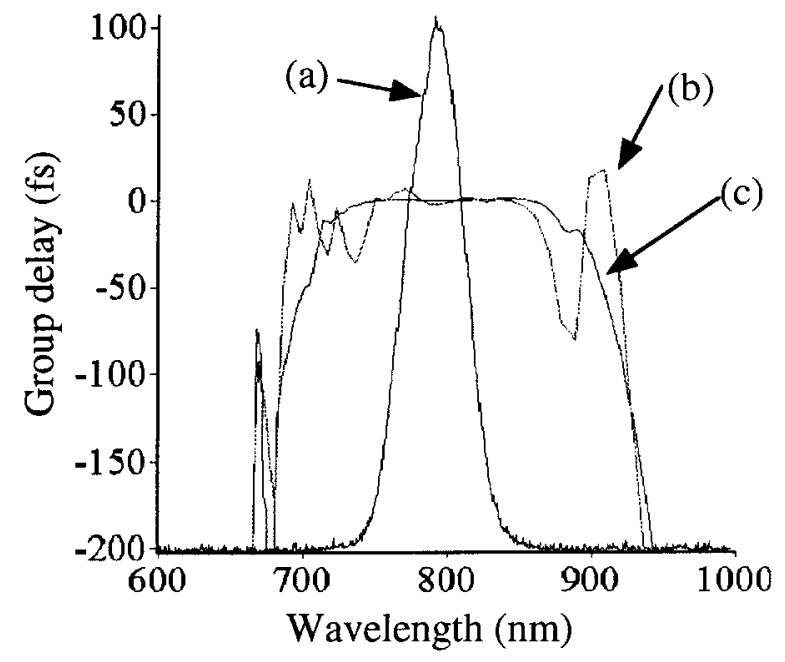

Fig. 18. Measured power spectrum (a) and group delay (b) along with computed group delay (c) for a high-energy $20 \mathrm{fs}$ pulse

teristically double-peaked, and were in excellent agreement with our model predictions (see Fig. 5), lending validity to our theory of higher-order dispersion-limited pulse formation in Ti:sapphire lasers. However, an alternative model of the double-peaked spectra suggested that the double-peaked spectrum was the result of coherent ringing in the laser medium [85]. Although both theories predict nearly identical pulse spectra and autocorrelations, the two theories predicted very different pulse shapes and shaping mechanisms. However, FROG can easily distinguish between the two different theories. Figure 17a,b shows the SHG FROG traces for the two theoretically predicted pulses, while Fig. 17c shows the experimentally measured FROG trace. The experimentally observed FROG trace agrees much better with that predicted by our high-order dispersion-limited theory [10] and therefore proves that the Ti:sapphire laser is 4OD limited at 7.5-9 fs. This result also illustrates the limitations of pulse measurement techniques based on autocorrelation fits - the theory of [85] was supported by an excellent-looking fit to interferometric autocorrelation data, where the fit assumes the pulse shape which FROG clearly shows is incorrect.

A second very new type of FROG, transient-grating frequency-resolved optical gating (TG-FROG) [86], has proven exceptionally useful for characterizing the output of our ultrafast amplifier systems [24,70]. Figure 18 shows the power of this FROG technique by comparing the measured and calculated group delay and spectrum from an ultrashortpulse amplifier system (shown in Fig. 14). This is the first time that FROG techniques have been used to characterize such ultrashort high-power pulses. The measured pulse duration is $20 \mathrm{fs}$, and the shape of the group delay curve indicates that the pulse duration is limited only by fifthorder dispersion. It should be noted that if a Gaussian pulse shape were assumed in an autocorrelation, the pulse width would be incorrectly determined to be $18 \mathrm{fs}$, while by assuming a $\operatorname{sech}^{2}$ pulse profile, the pulse width would be severely underestimated at $16 \mathrm{fs}$.

To design our amplifier systems, we developed a model that propagates the stretched pulse through the optical system, and predicts the output pulse characteristics while including the effects of gain narrowing, gain saturation, spectral shaping, dispersion, and gain dispersion. Figure 18 compares the model predictions to the experimental data for the output spectrum and group delay. There is excellent agreement between theory and experiment. The group delay is flat over the entire spectrum, except at the very edge where there is a slight distortion due to a small amount of residual fifth-order dispersion. It should be noted that this degree of agreement was made possible only with the phase information provided by FROG, which allowed us to identify and either correct or model sources of dispersion such as from the dielectric mirrors used in the system. FROG has proven to be very effective as an advanced diagnostic to help fine-tune dispersion compensation in the re-compression process. Very recently, we also used TG FROG to measure the shortest, high-power pulses generated to date. These pulses were generated by selfphase modulation (SPM) of our $20 \mathrm{fs}, 3 \mathrm{~mJ}$, pulses either in air [23] or in a hollow-core fiber [57]. Pulses of duration $9 \mathrm{fs}$ at a few microjoules of energy were obtained (as shown in Fig. 19), as well as millijoule-energy pulses at $13.5 \mathrm{fs}$. In the former case, an argon-filled hollow-core fiber $\sim 1$ meter long was used for SPM, with compression using a grating pair. In the latter case, SPM was accomplished by focusing the pulse in air and compressing it using dispersive mirrors. The information derived from TG FROG measurements allowed us to optimize the SPM process and the recompression of the SPM-generated light.

Recently, in collaboration with J. Peatross, we have also explored a deconvolution technique, called Temporal Information Via Intensity (TIVI) [87], which can rapidly obtain pulse amplitude and phase information from autocorrelation 


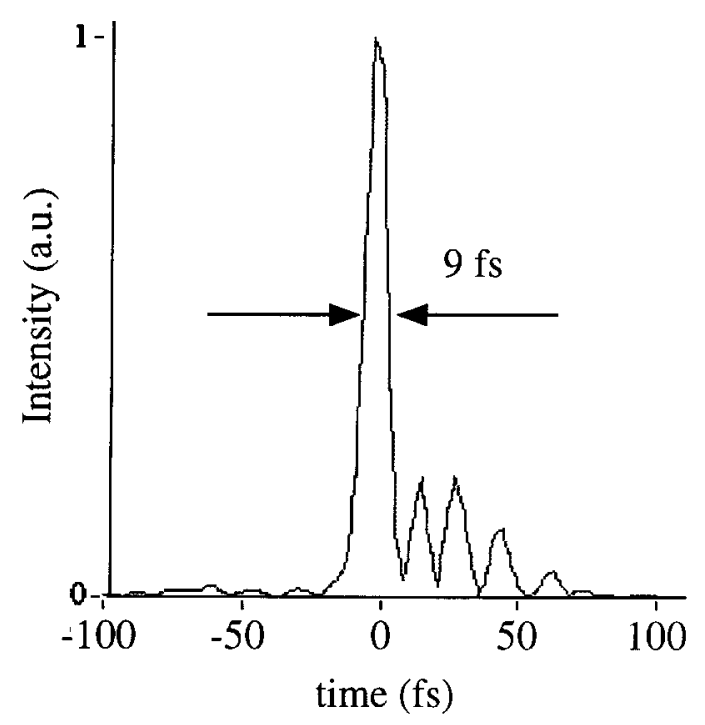

Fig. 19. 9 fs pulse generated using a hollow-core fiber compressor, measured using TG-FROG

and spectrum measurements. This method splits the amplitude and the phase determination into two discrete steps. Using the known positivity constraint on the second order autocorrelation, the first step uses a one-dimensional iterative loop which converges to the temporal pulse shape (with the usual temporal ambiguities inherent in second order measurements). This result, along with the measured power spectrum of the pulse, is used in the second half of TIVI, which applies a one dimensional Gerchberg/Saxton algorithm [88] to converge to the temporal phase. This method does very well on noise free data and is still very useful with up to $10 \%$ additive noise. However, it does suffer from the limitation, inherent to autocorrelation fit techniques, that the fit can stagnate to a qualitatively incorrect pulse shape, which yields a spectrum and autocorrelation that visually appear identical to the measured data. Nevertheless, this technique, when used in conjunction with full-blown FROG, can yield accurate deconvolutions with dramatically reduced computation time compared to FROG alone.

\section{Applications}

As discussed in the introduction, the interaction of intense light with matter can make possible many new phenomena of fundamental as well as practical interest, such as laserbased particle acceleration, new concepts in inertial confinement fusion, and ultrafast ultrashort-wavelength X-ray lasers. We have been pursuing the use of ultrashort amplified pulses to generate X-ray pulses with duration of only a few femtoseconds $[27,34,73,74]$. These ultrafast, coherent, soft-Xray pulses are produced simply by focusing a high-intensity femtosecond laser pulse onto a gas. The highly nonlinear interaction of the laser light with the atoms results in the emission of coherent high-order harmonics of the laser in the forward direction $[25,26,89]$. This process thus up-shifts a femtosecond pulse from the visible into the soft-X-ray region of the spectrum.

Coherent X-ray emission via high-harmonic generation can be understood to a first approximation using a semi-

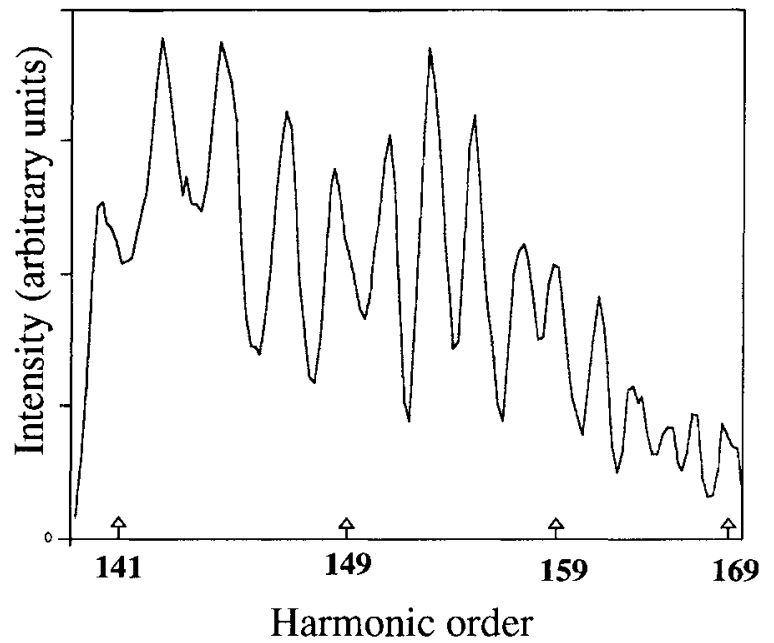

Fig. 20. Harmonics generated using a 25 fs pulse focused into Helium gas. The highest harmonic detected was the 169th of our $800 \mathrm{~nm}$ fundamental wavelength, or $47 \AA$

classical theory for the motion of an ionized electron during the first optical cycle after it is ionized due to the strong laser field $[90,91]$. In this picture, the highest photon energy achievable is determined by the maximum kinetic energy of this electron as it returns to the nucleus: $h v=I_{\mathrm{p}}+3.2 U_{\mathrm{p}}$, where $U_{\mathrm{p}}$ is the ponderomotive energy, and $I_{\mathrm{p}}$ is the ionization potential of the atom. $U_{\mathrm{p}}$ is determined by the energy gained by the electron in the laser field, which is in turn determined by the laser intensity at which the atom fully ionizes. We showed that, using very short-duration (26 fs) driving pulses, it is possible to generate photon energies substantially higher than with longer $100 \mathrm{fs}$ pulses from a given atomic gas. This is because the atom fully ionizes at a higher laser intensity for shorter duration excitation pulses, and the electron then gains more energy in the laser field (higher $U_{\mathrm{p}}$ ), which in turn results in higher radiated harmonic energies. Experimentally, we also demonstrated that shorter pulses generate harmonics more efficiently, and that it is possible to tune the wavelength of the harmonics by adjusting the chirp of the driving pulse.

In our most recent work, we generated the shortestwavelength femtosecond X-ray pulses to date. By focusing a $4 \mathrm{~mJ}, 26 \mathrm{fs}$ pulse into a helium gas jet, we generated harmonics down to a wavelength of $4.7 \mathrm{~nm}$, as shown in Fig. 20 [74]. This wavelength is very close to the "water window" region, where good contrast between water and carbon X-ray absorption is obtained. Moreover, our calculations show that the pulse duration of the highest harmonics are 1-3 fs, since they are generated only on the rising edge of the excitation pulse. Given the modest energies needed to generate these X-rays, it is now possible to produce very compact sources of femtosecond X-rays, which can be used for time-resolved X-ray studies of organic molecules.

More detailed calculations have determined that by using very short excitation pulses to drive high-harmonic emission, we are just beginning to enter a new non-adiabatic regime, where the response of the system depends on the time-history (pulse shape) of the excitation [73]. For even shorter excitation pulses, a new regime of harmonic generation by a "single-cycle" of the driver pulse can be reached [34, 35]. In 
this regime, the temporal coherence of the adjacent harmonic orders is dramatically improved compared with longer excitation pulses, even though the discrete harmonic structure in the emission disappears. X-ray pulses as short as 100 attoseconds can be emitted, with increased conversion efficiency of laser-to-harmonic radiation.

\section{Conclusions}

A new generation of laser sources has greatly enhanced our capability to generate and manipulate light, with pulse duration of only a few optical cycles, and with peak power and energy density achievable by few other methods on earth. We can also characterize and manipulate these pulses with unprecedented precision. This work has resulted and will continue to result in the observation of new physical phenomena and in new applications of such light sources.

Acknowledgements. The authors gratefully acknowledge support for their work from the National Science Foundation and the Department of Energy. H. Kapteyn acknowledges generous support from an Alfred P. Sloan Foundation Fellowship.

\section{References}

1. D.E. Spence, P.N. Kean, W. Sibbett: Opt. Lett. 16, 42 (1991)

2. H.C. Kapteyn, M.M. Murnane: Opt. Photon. News 5, 20 (1994)

3. M.T. Asaki, C.P. Huang, D. Garvey, J. Zhou, H.C. Kapteyn, M.M. Murnane: Opt. Lett. 18, 977 (1993)

4. P. Curley, C. Spielmann, T. Brabec, F. Krausz, E. Wintner, A. Schmidt: Opt. Lett. 18, 54 (1993)

5. J. Zhou, G. Taft, C.P. Huang, M.M. Murnane, H.C. Kapteyn, I. Christov Opt. Lett. 19, 1149 (1994)

6. A. Stingl, M. Lenzner, C. Spielmann, F. Krausz, R. Sipocs: Opt. Lett. 19, 204 (1994)

7. I.P. Christov, V. Stoev, M. Murnane, H. Kapteyn: Opt. Lett. 21, 1493 (1996)

8. I.D. Jung, F.X. Kärtner, N. Matuschek, D.H. Sutter, F. Morier-Genoud, G. Zhang, U. Keller, V. Scheuer, M. Tilsch, T. Tschudi: Opt. Lett. submitted (1997)

9. K.W. DeLong, R. Trebino, D.J. Kane: J. Opt. Soc. Am. B 11, 1595 (1994)

10. G. Taft, A. Rundquist, H.C. Kapteyn, M.M. Murnane, K. DeLong, R. Trebino, I.P. Christov: Opt. Lett. 20, 743 (1995)

11. G. Taft, A. Rundquist, H.C. Kapteyn, I.P. Christov, M.M. Murnane, K. DeLong, D. Fittinghoff, M. Krumbugel, R. Trebino: J. Quant. Electron. 2, 575 (1997)

12. D. Strickland, G. Mourou: Opt. Commun. 56, 219 (1985)

13. J.P. Zhou, C.P. Huang, C. Shi, H.C. Kapteyn, M.M. Murnane: Opt. Lett. 19, 126 (1994)

14. J.P. Zhou, C.P. Huang, M.M. Murnane, H.C. Kapteyn: Opt. Lett. 20, 64 (1995)

15. S. Backus, C. Durfee, M.M. Murnane, H.C. Kapteyn: Rev. Sci. Instrum., to be published (1997)

16. J.P. Chambaret, C. LeBlanc, G. Cheriaux, P. Curley, G. Darpentigny, P. Rousseau, G. Hamoniaux, A. Antonetti, F. Salin: Opt. Lett. 21, 1921 (1996)

17. B.C. Stuart, M.D. Perry, J. Miller, G. Tietbohl, S. Herman, J.A. Britten, C. Brown, D. Pennington, V. Yanovsky, K. Wharton: Opt. Lett. 22, 242 (1997)

18. A. Sullivan, H. Hamster, H.C. Kapteyn, S. Gordon, W. White, H. Nathel, R.J. Blair, R.W. Falcone: Opt. Lett. 16, 1406 (1991)

19. C. Barty, T. Guo C. Le Blanc, F. Raksi, C. Rose-Petruck, J. Squier, K. Wilson, V. Yakovlev, K. Yamakawa: Opt. Lett. 21, 668 (1996)

20. C.P.J. Barty, C.L. Gordon III, B.E. Lemoff: Opt. Lett. 19, 1442 (1994)

21. M. Pessot, J. Squier, G. Mourou, D.J. Harter: Opt. Lett. 14, 797 (1989)

22. F. Salin, J. Squier, G. Mourou, G. Vaillancourt: Opt. Lett. 16, 1964 (1991)
23. S. Backus, J. Peatross, C.P. Huang, M.M. Murnane, H.C. Kapteyn: Opt. Lett. 20, 2000 (1995)

24. S. Backus, C. Durfee, M.M. Murnane, H.C. Kapteyn: Opt. Lett., to be published (1997)

25. A. McPherson, G. Gibson, H. Jara, U. Johann, T.S. Luk, I.A. McIntyre, K. Boyer, C.K. Rhodes: J. Opt. Soc. Am B 4, 595 (1987)

26. A. L'Huillier, P. Balcou: Phys. Rev. Lett. 70, 774 (1993)

27. J. Zhou, J. Peatross, M.M. Murnane, H.C. Kapteyn, I.P. Christov: Phys. Rev. Lett. 76, 752 (1996)

28. S. Backus, J. Peatross, M. Murnane, H. Kapteyn: Opt. Lett. 21, 665 (1996)

29. M.M. Murnane, H.C. Kapteyn, M.D. Rosen, R.W. Falcone: Science 251, 531 (1991)

30. R.W. Schoenlein, W. Leemans, A. Chin, P. Volfbeyn, T. Glover, P. Balling, M. Zolotorev, K.-J. Kim, S. Chattopadhyay, C. Shank: Science 274, 236 (1996)

31. S.P. LeBlanc, M.C. Downer, R. Wagner, S.-Y. Chen, A. Maksimchuk, G. Mourou, D. Umstadter: Phys. Rev. Lett. 77, 5381 (1996)

32. D. Umstadter, J. Kim, E. Dodd: Phys. Rev. Lett 76, 2073 (1996)

33. J.H. Glownia, D.R. Gnass, P.P. Sorokin: J. Opt. Soc. Am. B 11, 2427 (1994)

34. I.P. Christov, M.M. Murnane, H.C. Kapteyn: Phys. Rev. Lett. 78, 1251 (1997)

35. P.B. Corkum, N.H. Burnett, M.Y. Ivanov: Opt. Lett. 19, 1870 (1994)

36. K. Schafer, K. Kulander: Phys. Rev. Lett. 78, 638 (1997)

37. P.F. Moulton: J. Opt. Soc. Am. B 3, 125 (1986)

38. P. Moulton: Proc. IEEE 80, 348 (1992)

39. J.A. Valdmanis, R.L. Fork: IEEE J. Quant. Electron, QE-22, 112 (1986)

40. C.P. Huang, H.C. Kapteyn, J.W. McIntosh, M.M. Murnane: Opt. Lett. 17, $139(1992)$

41. C.P. Huang, M.T. Asaki, S. Backus, M.M. Murnane, H.C. Kapteyn, H. Nathel: Opt. Lett. 17, 1289 (1992)

42. I.P. Christov, M.M. Murnane, H.C. Kapteyn, J.P. Zhou, C.P. Huang: Opt. Lett. 19, 1465 (1994)

43. I.P. Christov, H.C. Kapteyn, M.M. Murnane, C.P. Huang, J.P. Zhou: Opt. Lett. 20, 309 (1995)

44. I.P. Christov, V. Stoev, M. Murnane, H. Kapteyn: Opt. Lett. 20, 2111 (1995)

45. H.C. Kapteyn, M.M. Murnane: IEEE Leos Newsletter, August (1995)

46. L. Xu, C. Spielmann, F. Krausz, R. Sipocs: Opt. Lett. 21, 1259 (1996)

47. A. Stingl, M. Lenzner, C. Spielmann, F. Krausz, R. Sipocs, Opt. Lett. 20, 602 (1995)

48. B. Proctor, F. Wise: Appl. Phys. Lett. 62, 470 (1993)

49. F. Krausz, C. Spielmann, T. Brabec, E. Wintner, A.J. Schmidt: Opt. Lett. 17, 204 (1992)

50. H.A. Haus, J.G. Fujimoto, E.P. Ippen: IEEE J. Quant. Electron. 28, 2086 (1992)

51. H.A. Haus, J.D. Moores, L.E. Nelson: Opt. Lett. 18, 51 (1993)

52. Schott-Glass,

53. E.G. Lariontsev, V.N. Serkin: Sov. J. Quan. Electron. 5, 796 (1976)

54. J. Herrmann: J. Opt. Soc. Am. 11, 498 (1994)

55. G.P. Agrawal: Nonlinear Fiber Optics, Academic Press, (New York 1989)

56. O.E. Martinez, R.L. Fork, J.P. Gordon: Opt. Lett. 9, 156 (1984)

57. H. Avramopoulos, R.L. Fork: J. Opt. Soc. Am. B 8, 117 (1991)

58. M. Piche, J.F. Cormier, X. Zhu: Opt. Lett. 21, 845 (1996)

59. I.P. Christov, H.C. Kapteyn, M.M. Murnane: Opt. Lett., submitted (1997)

60. C. Barty, G. Korn, F. Raksi, C. Rose-Petruck, J. Squier, A. Tian, K. Wilson, V. Yakovlev, K. Yamakawa: Opt. Lett. 21, 219 (1996)

61. M. Lenzner, C. Spielmann, E. Wintner, F. Krausz, A.J. Schmidt: Opt. Lett. 20, 1397 (1995)

62. M. Nisoli, S.D. Silvestri, O. Svelto: Appl. Phys. Lett. 68, 2793 (1996)

63. F. Patterson, R. Gonzales, M. Perry: Opt. Lett. 16, 1107 (1991)

64. C.P.J. Barty, C.L.G. III, B.E. Lemoff: Opt. Lett. 19, 1442 (1994)

65. W.E. White, F.G. Patterson, R.L. Combs, D.F. Price, R.L. Shepherd: Opt. Lett. 18, 1343 (1993)

66. S. Kane: $\mathrm{PhD}$ thesis, University of Michigan (1996)

67. A. Weiner: Prog. Quant. Electr. 19, 161 (1995)

68. M.M. Wefers, K.A. Nelson: Science 262, 1381 (1993)

69. C.W. Hillegas, J.X. Tull, D. Goswami, D. Strickland, W.S. Warren: Opt. Lett. 19, 737 (1994)

70. C.G. Durfee, S. Backus, M.M. Murnane, H.C. Kapteyn: Opt. Lett., submitted (1997) 
71. W. Koechner: Solid-State Laser Engineering, Springer, (Heidelberg 1996)

72. A. DeFranzo, B. Pazol: Appl. Opt. 32, 2224 (1993)

73. I.P. Christov, J.P. Zhou, J. Peatross, A. Rundquist, M.M. Murnane, H.C. Kapteyn: Phys. Rev. Letter 77, 1743 (1996)

74. Z. Chang, A. Rundquist, H. Wang, M.M. Murnane, H.C. Kapteyn: Phys. Rev. Lett., submitted (1997)

75. D. Umstadter, S.-Y. Chen, A. Maksimchuk, G. Mourou, R. Wagner: Science 273, 472 (1996)

76. Bardeen, Wang, Shank: Phys. Rev. Letter 75, 3410 (1995)

77. Warren, Rabwitz, Dahleh: Science 259, 1581 (1993)

78. Kohler, Yakovlev, Che, Krause, Messina, Wilson, Schwntner, Whitnell: Phys. Rev. Lett. 74, 3360 (1995)

79. K.W. DeLong, R. Trebino, J. Hunter, W.E. White: J. Opt. Soc. Am. B 11, 2206 (1994)

80. K. Naganuma, K. Mogi, H. Yamada: IEEE J. Quant. Electr. 25, 1225
(1989)

81. V. Wong, I.A. Walmsley: J. Opt. Soc. Am. B 12, 1491 (1993)

82. J.R. Fienup: Appl. Opt. 21, 2758 (1982)

83. J.H. Seldin, J.R. Fienup: J. Opt. Soc. Am. A 7, 412 (1990)

84. D.R. Yankelevich, A. Knoesen, G. Taft, M.M. Murnane, H.C. Kapteyn, R.J. Twieg: Opt. Lett. 21, 1487 (1996)

85. J.D. Harvey, J.M. Dudley, P.F. Curley, C. Spielmann, F. Krausz: Opt. Lett. 21, 1487 (1994)

86. J. Sweetser, D. Fittinghoff, R. Trebino: Opt. Lett., submitted (1997)

87. J. Peatross, A. Rundquist: JOSA B, In preparation (1997)

88. R.W. Gerchberg, W.O. Saxton: Optik 35, 237 (1972)

89. J.J. Macklin, J.D. Kmetec, C.L. Gordon, III: Phys. Rev. Lett. 70, 766 (1993)

90. P.B. Corkum: Phys. Rev. Lett. 71, 1994 (1993)

91. J.L. Krause, K.J. Schafer, K.C. Kulander: Phys. Rev. Lett. 68, 3535 (1992) 